\title{
The Ionization Equilibrium of Iron in H II Regions
}

\author{
Rodríguez, M. \\ Instituto Nacional de Astrofísica, Óptica y Electrónica, INAOE, Apdo. \\ Postal 51 \& 216, Puebla, Pue 72000, Mexico \\ Rubin, R. H. \\ NASA Ames Research Center, Moffett Field, CA 94035-1000, USA
}

\begin{abstract}
We study the ionization equilibrium of Fe using photoionization models that incorporate improved values for the relevant atomic data. The previously available photoionization models predict concentrations of $\mathrm{Fe}^{3+}$ which are a factor of 3-8 higher than the values inferred from emission lines of [Fe III] and [Fe IV]. Our new models reduce these discrepancies to factors of $2-5$. We comment on the possible reasons behind the remaining discrepancies, and present an updated ionization correction factor for obtaining the $\mathrm{Fe}$ abundance from the $\mathrm{Fe}^{++}$abundance.
\end{abstract}

\section{Results}

We use the photoionization code NEBULA (Rubin et al. 1991a,b) updated with new ionization cross sections for $\mathrm{Fe}^{+}, \mathrm{Fe}^{++}, \mathrm{O}^{0}$ and $\mathrm{O}^{+}$, new recombination coefficients for $\mathrm{Fe}^{++}, \mathrm{Fe}^{3+}, \mathrm{O}^{+}$and $\mathrm{O}^{++}$, all the charge-exchange reactions involving these ions; and NLTE model stellar atmospheres (more details are given in Rodríguez \& Rubin 2003). Figure 1 shows the resulting ionization correction factors (ICFs), which imply:

$$
\frac{\mathrm{Fe}}{\mathrm{O}}=\mathrm{ICF} \frac{\mathrm{Fe}^{++}}{\mathrm{O}^{+}}=\frac{x\left(\mathrm{O}^{+}\right)}{x\left(\mathrm{Fe}^{++}\right)} \frac{\mathrm{Fe}^{++}}{\mathrm{O}^{+}}=\left(\frac{\mathrm{O}^{+}}{\mathrm{O}^{++}}\right)^{0.09} \frac{\mathrm{Fe}^{++}}{\mathrm{O}^{+}}
$$

where $x\left(X^{n+}\right)$ stands for the ionization fraction of the $X^{n+}$ ion. Figure 1 also shows a comparison with the ICFs implied by previous ionization models and with the results obtained by Rodríguez (2003) for several objects with available measurements of [Fe IV] lines. The new model results, although still higher than the measured values, are much closer to them. The remaining discrepancies translate into uncertainties of up to a factor of 4 in the Fe abundances derived for a wide range of objects, from the nearby Orion nebula to the low metallicity blue compact galaxy SBS 0335-052. Thus, resolving this problem has important implications for our understanding of both the evolution of dust in $\mathrm{H}$ II regions and the chemical history of low metallicity dwarf galaxies. These remaining discrepancies could be due to: (1) the need for further improvements in the ionization models, (2) errors in the atomic data (in particular the colli- 
sion strengths) used to derive the $\mathrm{Fe}^{++}$and $\mathrm{Fe}^{3+}$ abundances (see Rodríguez \& Rubin 2003 for a further discussion of these issues).

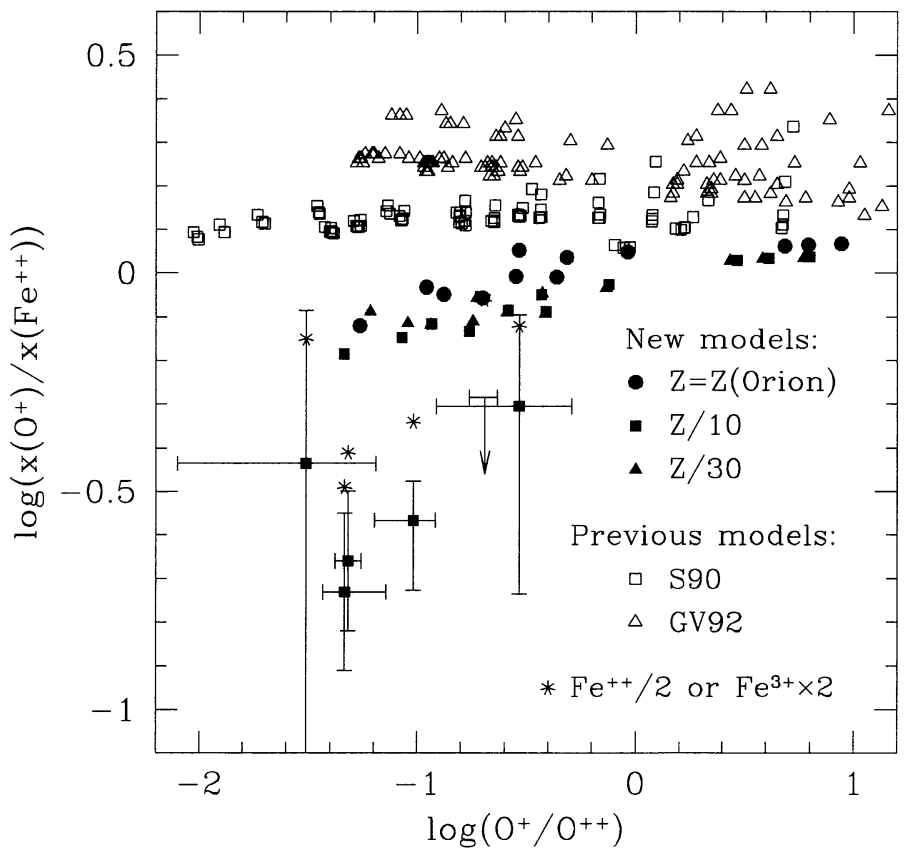

Figure 1. ICFs obtained from our new models compared with the previous model results of Stasińska (1990, S90) and Gruenwald \& Viegas (1992, GV92). The squares with error bars show the results obtained in Rodríguez (2003) for objects with available measurements of [Fe IV] lines: from left to right, the planetary nebula IC4846, SMC N88A (position 1), SBS 0335-052, SMC N88A (position 2), 30 Doradus (an upper limit), and M42 (the Orion nebula).

Acknowledgments. Project partially supported by the Mexican CONACYT project J37680-E. RR acknowledges support from the NASA Long-Term Space Astrophysics program.

\section{References}

Gruenwald, R.B., \& Viegas, S.M. 1992, ApJS, 78, 153 (GV92)

Rodríguez, M. 2003, ApJ, 590, 296

Rodríguez, M., \& Rubin, R.H. 2003, astro-ph/0312246

Rubin, R.H., Simpson, J.P., Haas, M.R., \& Erickson, E.F. 1991a, ApJ, 374, 564

Rubin, R.H., Simpson, J.P., Haas, M.R., \& Erickson, E.F. 1991b, PASP, 103, 834

Stasińska, G. 1990, A\&AS, 83, 501 (S90) 